\title{
SEXTANT X-ray Pulsar Navigation Demonstration: Flight System and Test Results ${ }^{1}$
}

\author{
Luke M. B. Winternitz ${ }^{2}$, Jason W. Mitchell, Munther A. Hassouneh, Jennifer E. Valdez, Samuel R. Price \\ Sean R. Semper, Wayne H. Yu \\ NASA Goddard Space Flight Center \\ Greenbelt, MD 20771, USA \\ Paul S. Ray, Kent S. Wood \\ Naval Research Laboratory \\ Washington, DC 20375, USA

Zaven Arzoumanian
NASA GSFC (USRA)
Greenbelt, MD 20771, USA \\ Keith C. Gendreau \\ NASA GSFC, Code 662 \\ Greenbelt, MD 20771, USA
}

\begin{abstract}
The Station Explorer for X-ray Timing and Navigation Technology (SEXTANT) is a technology demonstration enhancement to the Neutron-star Interior Composition Explorer (NICER) mission. NICER is a NASA Explorer Mission of Opportunity that will be hosted on the International Space Station (ISS). SEXTANT will, for the first time, demonstrate real-time, on-board X-ray Pulsar Navigation (XNAV), a significant milestone in the quest to establish a GPS-like navigation capability available throughout our Solar System and beyond.
\end{abstract}

This paper gives an overview of the SEXTANT system architecture and describes progress prior to environmental testing of the NICER flight instrument. It provides descriptions and development status of the SEXTANT flight software and ground system, as well as detailed description and results from the flight software functional and performance testing within the highfidelity Goddard Space Flight Center (GSFC) X-ray Navigation Laboratory Testbed (GXLT) software and hardware simulation environment. Hardware-in-the-loop simulation results are presented, using the engineering model of the NICER timing electronics and the GXLT pulsar simulator-the GXLT precisely controls NASA GSFC's unique Modulated X-ray Source to produce $X$-rays that make the NICER detector electronics appear as if they were aboard the ISS viewing a sequence of millisecond pulsars.

SEXTANT is funded by the NASA Space Technology Mission Directorate, and NICER is funded by the NASA Science Mission Directorate.

\section{TABLE OF CONTENTS}

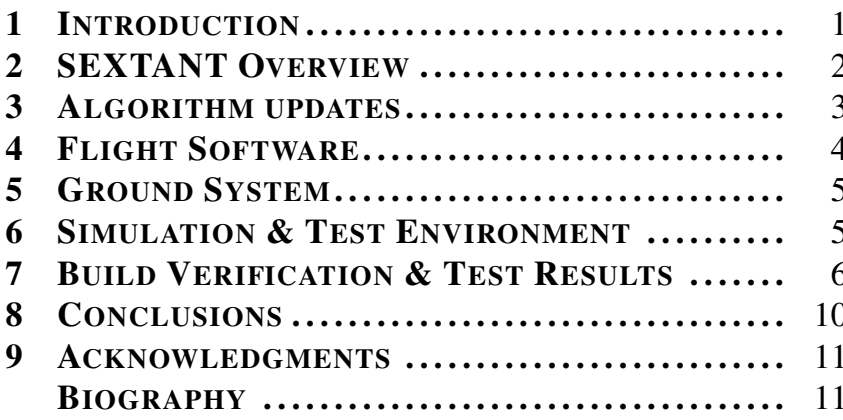

\section{INTRODUCTION}

NASA's Station Explorer for X-ray Timing and Navigation Technology (SEXTANT) project [1] will provide the first

\footnotetext{
${ }^{1}$ This is a work of the U.S. Government and is not subject to copyright protection in the United States.

${ }^{2}$ Corresponding author, luke.b.winternitz@nasa.gov
}

demonstration of autonomous spacecraft navigation using X-ray Pulsars as beacons. This concept, often referred to as XNAV, has the potential to provide a GPS-like autonomous navigation capability that works throughout the Solar System, serving as a complement and navigation backup to NASA's Deep Space Network (DSN), and enabling exploration beyond the Solar System.

XNAV exploits the atomic clock-like stability of faint pulsations from a class of rapidly rotating neutron stars known as Millisecond Pulsar (MSP). This stability allows long-term predictions of pulse phase, relevant to a reference location, to be generated and stored in a catalog. Measurements of MSP pulse phase made on-board the spacecraft with an XNAV sensor can then be compared to the catalog predictions to obtain information about the user's position relative to the reference location. The concept of navigating via pulsars, and in particular XNAV, has a long history of development dating back to the discovery of the first radio pulsar in the 1960's. A concise review of past research and development is provided in [2].

SEXTANT is a technology enhancement to the Neutron-star Interior Composition Explorer (NICER) mission, which is an X-ray Astrophysics Mission of Opportunity to the International Space Station (ISS) that will undertake a fundamental investigation of extremes in gravity, material density, and electromagnetic fields of rapidly spinning neutron stars via time-resolved X-ray spectroscopy [3, 4]. NICER and SEXTANT are funded through a cost-sharing opportunity between the NASA Science Mission Directorate and NASA Space Technology Mission Directorate Game Changing Development Program Office.

NICER will achieve its science objectives by deploying an $\mathrm{X}$-ray telescope instrument, shown in Figure 1, as an attached payload on a zenith-side ExPRESS Logistics Carrier aboard the ISS. The instrument consists of 56 pairs of X-ray Concentrators and Silicon Drift Detectors attached to and aligned within the Integrated Optical Bench. Each photon interacting with a detector is assigned an energy estimate and a timestamp. NICER offers over an order-of-magnitude improvement in time-coherent sensitivity and timing resolution beyond the capabilities of any X-ray observatory flown to date. The large effective collecting area results in a high signal-to-background ratio, which together with its precise timing, make NICER's instrument a nearly ideal XNAV sensor. SEXTANT will use a subset of the data collected for the NICER science program, on-board and in real-time, to demonstrate X-ray pulsar navigation in the highly dynamic, low-Earth orbit regime. The primary Key Performance Parameter (KPP) of the technology demonstration is to maintain 


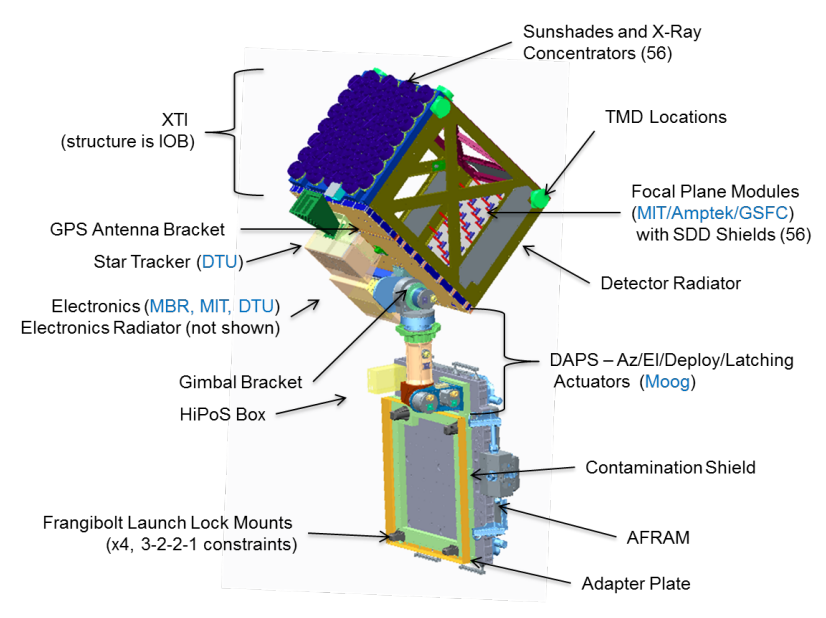

Figure 1. NICER payload mechanical model.

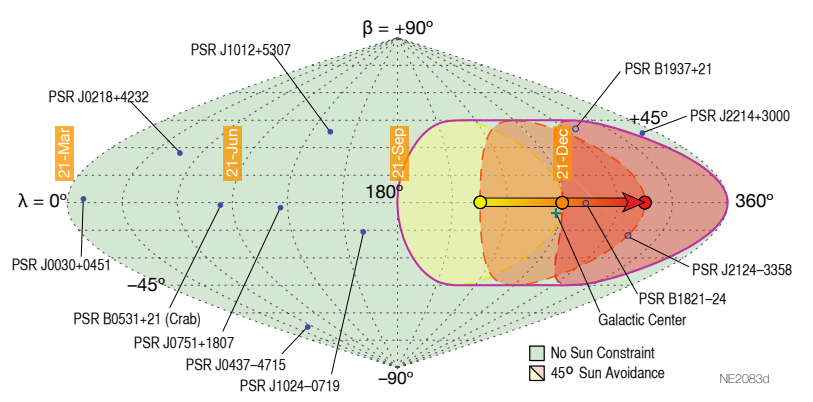

Figure 2. SEXTANT MSP target locations, in ecliptic coordinates $(\lambda, \beta)$, including the 3 month effect of a $45^{\circ}$ Sun avoidance constraint, centered on the Winter Solstice.

orbital position knowledge within $10 \mathrm{~km}$, worst direction, using up to 2 weeks of MSP observations.

In this paper, we will review the SEXTANT architecture and describe recent progress for both NICER and SEXTANT. Next, we briefly describe updates to the SEXTANT algorithms as presented in [2]. Then, we provide details on the SEXTANT flight system-consisting of the on-board component, termed the X-ray Pulsar Navigation Flight Software (XFSW), and the ground component, called the X-ray Pulsar Navigation Ground Segment/System (XGS)-and briefly review the GSFC X-ray Navigation Laboratory Testbed (GXLT). Next, we describe the test regime within the GXLT used to verify the SEXTANT flight system, and provide recent test results. Finally, we describe the path forward and give concluding remarks.

\section{SEXTANT OVERVIEW}

In this section we review the SEXTANT system architecture and provide brief status updates for NICER and SEXTANT.

\section{SEXTANT System Architecture}

Figure 3 provides a graphical overview of the SEXTANT system architecture showing the four main components. During the SEXTANT experiment(s), the X-ray Timing Instrument (XTI) points sequentially at suitable navigation MSP targets, shown in Figure 2, and provides X-ray photon event times, accurate within $100 \mathrm{~ns}$ (RMS) of GPS time, and an energy estimate for each event to the XFSW. The XFSW filters events based on energy and other criteria to reduce unwanted background events, thus increasing signal-to-noise ratio. Accepted event times are collected in a buffer along with a phase prediction at the event time derived from the pulsar model and a spacecraft state prediction provided by the Goddard Enhanced Onboard Navigation System (GEONS) Extended Kalman Filter (EKF), a key component of the XFSW [5, 6]. When a sufficient number of events from a pulsar are collected, those events are batch processed by a Maximum Likelihood (ML) estimator to extract point estimates of pulse phase and frequency. These estimates are passed as measurements to GEONS to update the orbit state estimate. The XGS maintains the pulsar almanac-the full timing models, pulse templates, count rates, and raw data used to derive all of these-and provides periodic updates to the XFSW configuration table including a subset of the pulsar almanac, sends asynchronous commands as needed, and monitors SEXTANT system health and status, as well as performance. Finally, all development and testing of the SEXTANT flight system takes place in the GXLT, a unique high-fidelity test environment able to provide stimulus to the flight software and hardware modeling SEXTANT or other XNAV scenarios.

A description of the SEXTANT system architecture is presented in [7], and a detailed overview of the full SEXTANT system design and testbed, with a focus on algorithms, is provided in [2].

\section{NICER status}

NICER is in Phase C [8] and progressing on-schedule. At this time, all major flight components have been delivered to National Aeronautics and Space Administration (NASA) GSFC, and the payload is being integrated and tested-a recent picture of the integrated flight XTI just before thermalvacuum testing is given in Figure 4. NICER will complete payload integration and pre-environmental functional testing in late 2015. Also in late 2015, NICER will have its preenvironmental review in preparation for Key Decision PointD. NICER is on-target for a June 2016 delivery to Cape Canaveral for launch via a SpaceX Commercial Resupply Services mission, no earlier than August 2016.

\section{SEXTANT development status and recent progress}

SEXTANT algorithms used in XFSW (and also run in parallel on the ground system) must deal with a broad dynamic range in count rates because the pulsars utilized have a corresponding large range in brightness. A previous paper [2] described in detail the algorithms for faint sources. This paper will only outline the algorithms approach in general but then will cover in detail the special case of the brighter sources, meaning accommodation for higher count rates. These algorithm augmentations have been incorporated in XFSW v2.1.0, and are described in $\S 3$.

The XFSW, described in $\S 4$, is nearing completion. XFSW v2.1.0 has been integrated into the most recent NICER Instrument Flight Software (IFSW) build, which is currently supporting payload level testing. An optional cleanup build may be completed this year, while the final XFSW version is planned for release in early 2016. XFSW v2.1.0 passed a comprehensive set of build verification tests that are described in detail in $\S 6$.

In September 2015, SEXTANT completed its annual Game Changing Development Program Office Continuation Review 


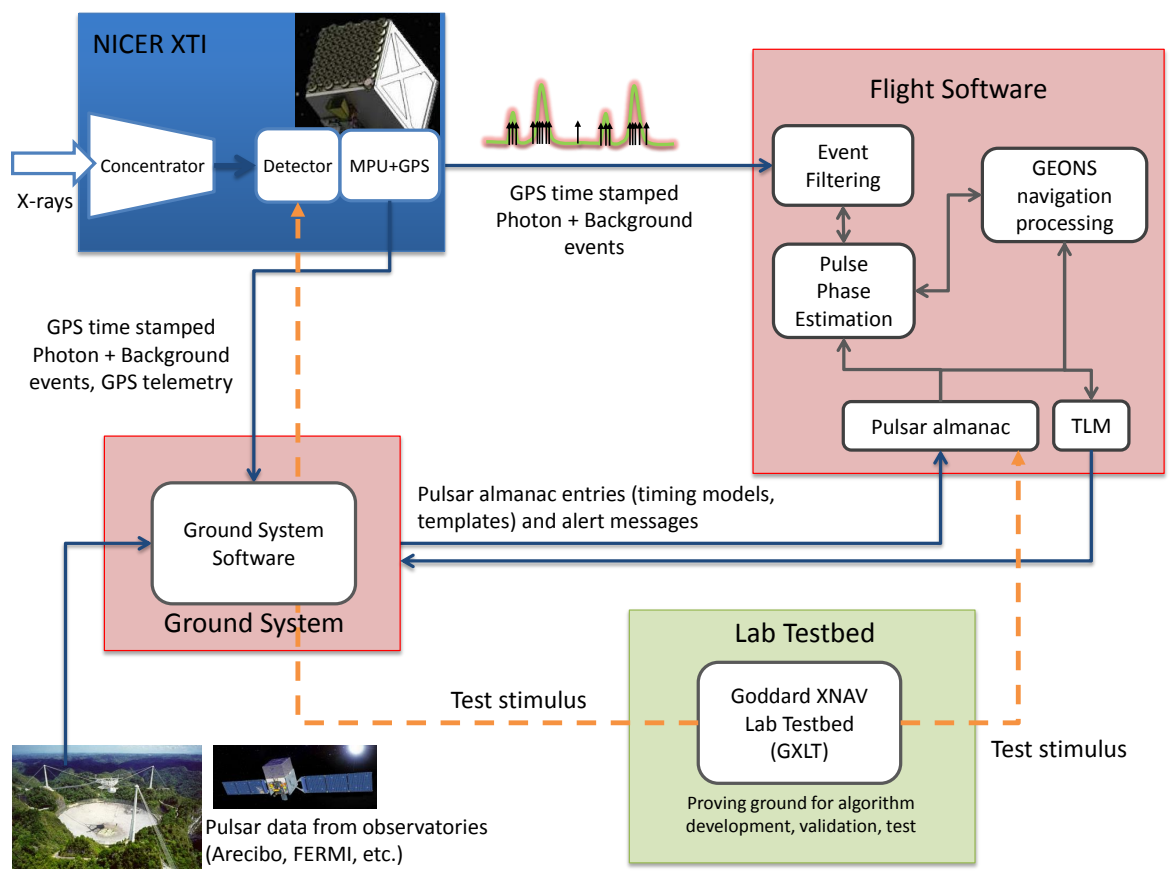

Figure 3. Block diagram of SEXTANT system architecture.

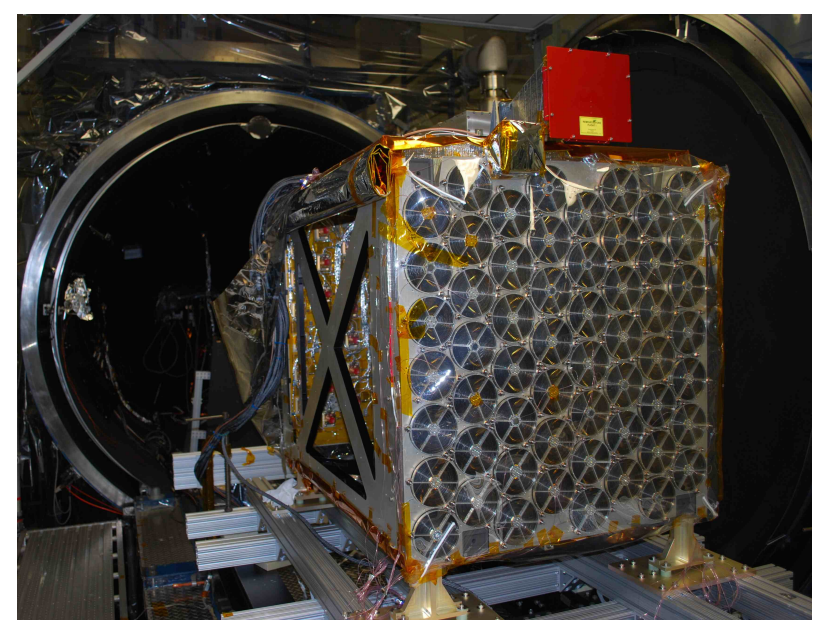

Figure 4. Photograph of the integrated NICER XTI about to begin thermal-vacuum testing.

for the following fiscal year, and remains on schedule to meet all planned milestones.

The XGS architecture and basic concept of operations is now well defined, and described briefly in $\S 5$. Moving forward, the SEXTANT team plans a major XGS build in late 2015 with a final build to support mission readiness testing in spring 2016.

Both the GXLT software and hardware simulation, described in detail in [2] and briefly in $\S 6$ below, have seen fidelity improvements. In particular, the hardware testbed has been updated to support testing with the NICER hardware timing chain, as well as NICER payload timing tests.

\section{Algorithm UPDATES}

Reference [2] provides a detailed description of the SEXTANT low-flux algorithms. Since the publication of that work, several updates have been integrated into the flight system. The most important of these are: a) the ability to process the high-flux Crab pulsar, and b) estimation of count rate parameters in addition to phase and frequency corrections.

The following approach is used to obtain final estimates of corrections to pulse phase and frequency predictions and signal and background count rates based on a batch of photon events.

1. Collect sufficiently many events, after filtering out background photons using available instrument- and pulsarspecific discriminators, such as photon energy and event location on the detector.

2. Obtain a coarse estimate of the unfiltered "background" rate (including any unmodulated emission from the pulsar) by subtracting the catalog value of the signal rate from the observation empirical total rate.

3. For low-flux MSPs, determine phase and frequency correction estimates by maximizing the likelihood (using the notation of [2])

$$
\begin{gathered}
l(\hat{\theta})=\sum_{k=1}^{N} \log \lambda\left(\tilde{\phi}\left(T_{k}\right)+q+f\left(T_{k}-t_{a}\right)\right) \\
-(\alpha+\beta)\left(t_{b}-t_{a}\right) .
\end{gathered}
$$

over a regular grid of phase and frequency corrections $(q, f)$. Here $\lambda$ is the rate function, and $\left\{\tilde{\phi}\left(T_{k}\right)\right\}_{k=1}^{N}$ are the phase predictions at each of $N$ event times $\left\{T_{k}\right\}_{k=1}^{N}$ collected over observation interval $\left[t_{a}, t_{b}\right]$. In this step, the signal $\alpha$ and background $\beta$ count rates are held constant. (For this reason, the second term in (1) was omitted in [2], but it will be varied in the following step.) 
The range of the grid is set according to the predicted phase and frequency measurement variance square-root provided by the EKF and the resolution of the grid is set to a multiple of the measurement noise root-variance.

4. Optionally, perform a configurable number of modified Newton iterations on the likelihood function (1), varying all parameters $(q, f, \alpha, \beta)$ and with initial iterate set to the grid search optimized $(q, f)$ of Step 3 and coarse count rate estimates $(\alpha, \beta)$ from Step 2 .

Reference [2] describes Step 3, in detail, for low-flux MSPs. In the following two sections, we provide a) an update to Step 3 for high-flux targets, e.g., the Crab pulsar, and b) a brief description of Step 4.

A phase-binned estimator for high-flux targets-The ML phase estimation algorithm described in [2] is expected to fit comfortably within the SEXTANT allocation for CPU utilization for all pulsars in the SEXTANT catalog, with the exception of the Crab pulsar, which has between 4-5 orders of magnitude higher flux.

For high-flux targets, we approximate the approach described above by quantizing the fractional predicted phase to the bin center $\left\{\bar{\phi}_{j}\right\}_{j=1}^{M}$ of one of $M$ (nominally equal to 1024) regularly spaced phase bins and counting the number $\left\{n_{j}\right\}_{j=1}^{M}$ of events that fall into each bin.

Because this pre-binning destroys the absolute (whole cycle) phase information, only a phase correction and no frequency correction is estimated, i.e., $f=0$. This is expected to be adequate over the much shorter observation intervals needed when processing the Crab, as compared to the other SEXTANT pulsars. (Typical observation times are given in [2].) This assumption makes the likelihood function independent of the length $N$ of the time of arrival sequence, yielding the approximation to (1), involving only $M \ll N$ terms, given as

$$
l(\hat{\theta}) \simeq \sum_{j=1}^{M} n_{j} \log \lambda\left(\bar{\phi}_{j}+q\right)-(\alpha+\beta)\left(t_{b}-t_{a}\right)
$$

Thus, for high-flux targets, (2) replaces (1) in Step 3 described above.

Newton iterations - In Step 4, we attempt to refine the grid optimized phase and frequency corrections of Step 3, the coarse background count rate estimate of Step 2 and the catalog value of the signal count rate using a continuous optimization approach. Specifically, an unconstrained maximization of (1) is attempted using a modified Newton approach to ensure a direction of descent and determine an appropriate step size. To compute the search direction, a modified Cholesky factorization [9] of the negative log-likelihood Hessian is attempted. If the factorization encounters a negative value on the diagonal, instead of failing, it is replaced by a configurable positive value. Once the search direction is computed, a line search is used to determine an adequate step size satisfying the Armijo conditions [9]. While this component has been implemented in the GXLT, it does not yet appear in the XFSW.

\section{Flight Software}

The SEXTANT X-ray Pulsar Navigation Flight Software (XFSW) is a GSFC Core Flight System (CFS) application. The CFS, described in [10], is GSFC's standard mission flight software framework that arranges core flight software units, e.g., data ingest, telemetry output, etc, and custom applications on a software message bus. Each CFS app subscribes to a relevant set of command and telemetry messages. The SEXTANT XFSW is a message driven CFS application responding to three external messages:

1. Detector photon packets, which provide the raw photon event data,

2. Pointing Control packets, which tell the XFSW which target it is looking at, and

3. GPS state packets, used to seed the EKF and used for onboard performance monitoring, as well as timing of the application as these packets arrive at a convenient $1 \mathrm{~Hz}$ rate.

The flow of the XFSW is shown in block diagram form in Figure 5. Initially, when the XFSW is commanded to start, and when appropriate conditions are met on the quality of the Global Positioning System (GPS) point-solution, a GPS state packet triggers a one-time initialization of the GEONS EKF. From that point forward, each tenth GPS packet triggers a nominal 10 second GEONS forward propagation of the state estimate, kept ahead of the incoming photon packet times by 30 seconds. The propagated state estimates are entered into a state buffer as they are generated. Photon event packets are passed through a photon filter to reduce background and out-of-band events. The propagated state buffer and pulsar timing models are used to assigned a received pulse phase and frequency prediction at each event time. This information is inserted into an event buffer assigned to the current pulsar. Once a buffer contains a threshold number of events, the ML batch processor operates on it to extract a point estimate of the phase and frequency (and count rates) of the current pulsar's pulsation. These estimates are passed as measurements into GEONS, triggering a measurement/state update. Pointing Control packets trigger an update to the current pulsar when they indicate a new SEXTANT target is being accurately tracked, or a switch to the "null pulsar" idle state when no target is being tracked.

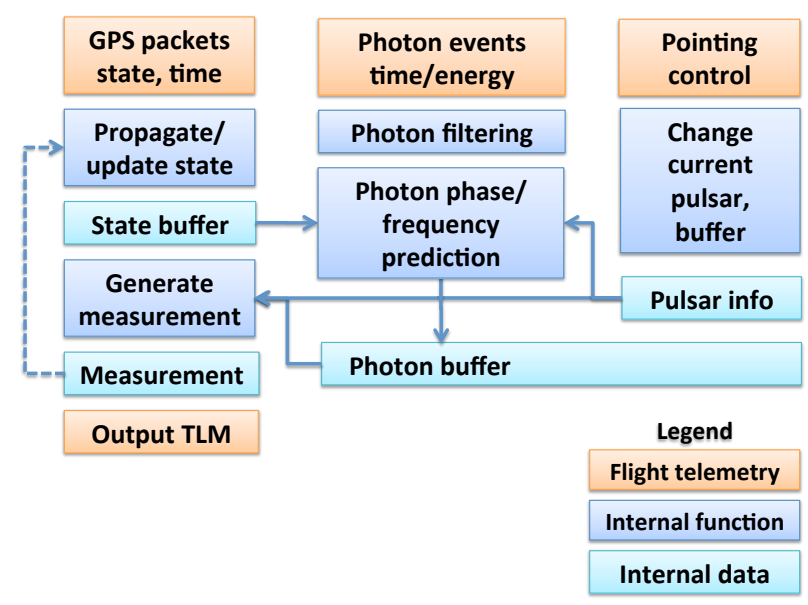

Figure 5. Message driven flow of the SEXTANT XFSW.

In the flow described above, the XFSW photon batch processing algorithms require the EKF's forward propagated state estimates to be sufficiently accurate. This is expected to 
be true during the SEXTANT primary experiment when the SEXTANT team determines the target schedule. During other times, when SEXTANT plans to operate, but does not control the pointing schedule, propagated state estimates based on XNAV measurements alone may not be sufficiently accurate for the batch processor. In this case, the XFSW will be commanded to switch to a calibration mode where pointsolution state estimates from the GPS packets are passed to the EKF to maintain filter convergence so that the event filtering, buffering, and ML batch processor can run. This mode will also be used in early mission operations, during which the pulse template models and count rate estimates will be refined using NICER data, and components of the XFSW will be checked out and reconfigured, if necessary, to optimize performance.

The XFSW is controlled through a configuration table and a handful of discrete commands, to start/stop/reset the application. The configuration table consists of the pulsar upload table (a compressed subset of the pulsar almanac including pulse templates, count rates, pulsar direction, and polynomial representations of the timing model), detector calibration tables, photon filtering and processing parameters, and GEONS filter configuration and data. As shown in Figure 3, the XGS is responsible for generating and uplinking the XFSW configuration table and supplying any discrete commands.

The full configuration table consists of about 300 kilobytes of data uncompressed, with an expected (compressed) upload size of 10 kilobytes requiring approximately two minutes to update. The XGS nominally will provide an update to the configuration table every three days, but this will typically consist of a small subset of the full configuration table.

\section{GROUND SYSTEM}

While the role of the XGS as described in [7] has not changed, the architecture and basic concept of operations has been clarified since that publication, and is described in further detail here.

The XGS must perform the following tasks.

- Generate and maintain the pulsar almanac.

- Maintain and update the XFSW configuration table information.

- Deliver and iterate on an observation schedule with the NICER ground system.

- Build and upload the XFSW configuration table according to NICER upload schedule on a nominal operations cadence.

- Monitor the performance of the SEXTANT XFSW.

- Generate and upload asynchronous commands to start, stop, and reset the XFSW or its components.

- Be able to run a copy of the XFSW on the ground using flight data, in order to verify XFSW results and run experimental improvements.

The XGS, shown at a top level in Figure 6, is divided into three primary components which reside in different physical locations and with distinct responsible parties. The pulsar almanac timing models, templates and count rate estimates are maintained and updated by SEXTANT Naval Research Laboratory (NRL) pulsar astrophysicist team members. The XFSW configuration update system is managed in the SEXTANT lab by GSFC SEXTANT team members, and the visibility analysis tool and interface to the flight system is controlled by the NICER operations team in the NICER Science Mission Operations Center (SMOC).

The basic concept of operations for the XGS is the execution of the following sequence of actions which occurs in synchrony with the nominal 3-day cadence of the NICER target schedule planning cycle (refer to Figure 6):

1. Ingest external information, including an ISS predictive ephemeris, Earth Orientation Parameters (EOP) and pulsar timing data from ground-based radio observatories and space-based observatories, especially NICER. ${ }^{2}$

2. Evaluate pulsar visibility as a function of time along the orbit prediction. If necessary, update pulsar almanac.

3. Generate a candidate observation schedule with the SEXTANT scheduling tool and manually set XFSW configuration parameters to tune performance.

4. Generate the XFSW configuration table.

5. Verify schedule and configuration in GXLT simulation. If verification fails, return to Step 3.

6. Send proposed observation schedule to NICER scheduling tool for approval and adjustment if needed, to obtain final schedule.

7. Regenerate the XFSW configuration table.

8. Verify NICER approved schedule in GXLT. If verification fails, return to Step 3.

9. Deliver final XFSW configuration table to NICER SMOC for upload.

\section{Simulation \& Test EnVironment}

We briefly describe the structure of the test environment here and refer the reader to [2] for details. Referring to Figure 7, we now walk through the GXLT simulation. The simulation starts with the definition of a XNAV scenario, specifying pulsar targets and models, initial spacecraft state and epoch, detector description and parameters, simulation algorithm and flow configuration. Next, a high-fidelity reference trajectory is generated and particle background rates for the detector along the trajectory are computed. For a simulation of SEXTANT on NICER, data from the ISS Miniature Array of Radiation Sensors (MARS) experiment [11] are used. Next, visibility to the target list is evaluated, accounting for celestial body and ISS structure occultation, and NICER hardware constraints. An optimized observation schedule is then generated that obeys these constraints. Truth phase and frequency time histories to each pulsar using high-fidelity pulse timing models are generated with radio observatory data and the TEMPO2 pulsar timing software [12]. At this point the simulation branches into three modes or "levels." At Level-0, simulated (Gaussian) errors with noise levels set appropriately for the detector and pulsar models are added to the truth phase and frequency observables. At Level-1, a nonhomogeneous Poisson process simulation is run to generate a file of photon event times covering the entire simulation that accurately model the phase history, pulsar template, and count rates. Finally at Level-2, the truth observable file is used to drive the GXLT hardware testbed which produces real-time $\mathrm{X}$-ray photon events with precisely controlled timing characteristics and pulse shape, with an energy spectrum in the passband of the NICER XTI, and optionally, synchronized Radio Frequency (RF) signals to stimulate an integrated GPS receiver.

\footnotetext{
${ }^{2}$ While an opportunity to ingest new EOPs, used by the GEONS filter, and pulsar timing data, used to maintain pulsar timing models, will exist nominally every three days, actual updates are expected to occur much less often.
} 


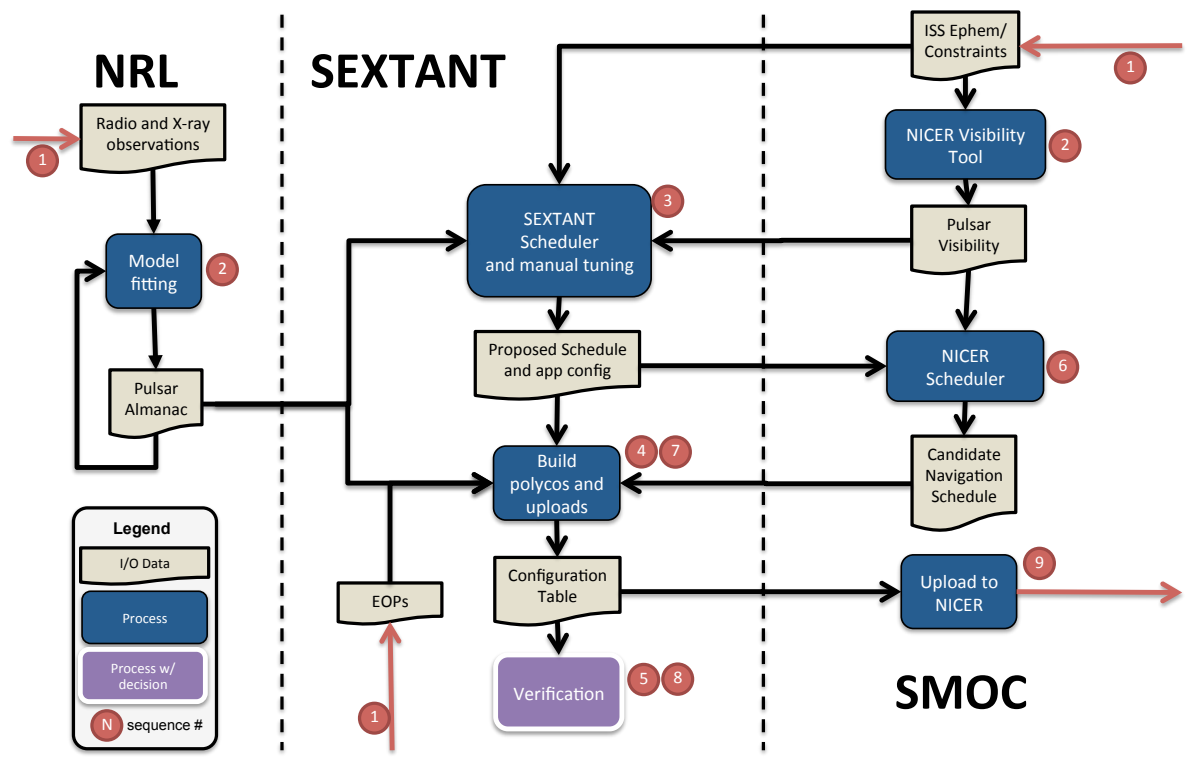

Figure 6. XGS top level architecture.

Thus, the simulation output is either a file of noise-corrupted pulse phase and frequency measurements, a file of photon times corrupted by Poisson noise, or real-time X-ray photon events and GPS RF stimulus that can be used to stimulate the NICER XTI Engineering Model (EM) or flight hardware.

The Level-0 file of noise corrupted phase and frequency measurements is useful for evaluation of long term XNAV scenarios and was used in early SEXTANT development. In [13], a study of a practical XNAV sensor along a Cassinilike cruise trajectory was modeled in the GXLT Level-0 simulation and performance was compared to DSN tracking using GSFC's Orbit Determination Toolbox (ODTBX) [14]. However, SEXTANT flight system development has relied primarily on Level-1 software simulated photons and Level-2 real-time X-ray stimulus for development and test.

\section{BUILd VERIFICATION \& TeSt Results}

Each XFSW release is subjected to a comprehensive build verification suite consisting of the following components:

- Baseline performance test using the GXLT Level-1 simulation,

- XFSW unit test of photon processing routines within GXLT Level-1 simulation,

- XFSW application test on PC driven by Python script,

- NICER IFSW integration test on the NICER Main Electronics Box (MEB) EM, again with GXLT Level-1 simulated events,

- Hardware system test on the MEB EM using full single NICER EM timing chain stimulated by the GXLT Level2 pulsar simulator.

In the following sections, we describe the structure and intent of each component and provide test results for the most recent build 2.1.0 of the XFSW released in August of 2015.

\section{XFSW v2.1.0 build test scenario}

XFSW v2.1.0 build verification testing relied on stimulus from the Level-1 and Level-2 outputs of the GXLT simulation. We briefly describe the GXLT scenario definition here.
A high-fidelity reference orbit was generated with a simulation epoch and initial state consistent with planned SEXTANT experiments. Pulsars B1821-24, B1937+21, and J0534+2200 (the Crab pulsar) ${ }^{3}$ were observed sequentially according to an observation schedule optimizing certain performance metrics, while obeying celestial body and ISS occultation and payload hardware constraints. Realistic, but non-flight, pulsar template and timing models were provided by NRL team members. Static signal and background count rates were based on current estimates of NICER XTI characteristics, while the variable particle background component was modeled on MARS sensor data [11]. For further discussion of SEXTANT count rate modeling, see [2]. The filter state was initialized using a state from the truth trajectory with noise added corresponding to Semi-Major Axis error with a $1 \mathrm{~km}$ bias and a $2 \mathrm{~km}$ random component, or from a similarly degraded GPS solution for the case of the Hardware System Test. The XFSW was loaded with a flight-like configuration table.

Besides the use of the fake Crab we note here some limitations of the XFSW v2.1.0 build verification test setup. First, while the reference orbit was generated with a different orbit propagator-the General Mission Analysis Tool (GMAT) [15] — using a higher-order geo-potential model and higherfidelity drag model than that used in the GEONS propagator, no additional errors were introduced into the spacecraft parameters (mass, area, center-of-mass offset, etc.). Similarly, the same pulsar models were used both in the GXLT simulation and by the XFSW. Finally, while the results presented have been observed to be typical, only a single arc was used to determine whether the test passed or failed. We plan to remedy these shortcomings in future work for inclusion in future build test scenarios and publications. In particular, work on tools to add realistic errors to the orbit and pulsar models provided to the estimator has begun, as has work on a Monte Carlo simulation that will vary model errors, photon simulation, and initial state errors.

\footnotetext{
${ }^{3}$ In this test, the Crab pulsar was artificially moved to a location in the sky where it was visible (namely to the coordinates of pulsar J0218+4232) because, in the nominal simulation period, the true Crab was occulted by a hardware keep-out cone around the Moon. We refer to this fictitious target as the fake Crab and designate it in test output as P SR_J0534+2200 fake.
} 


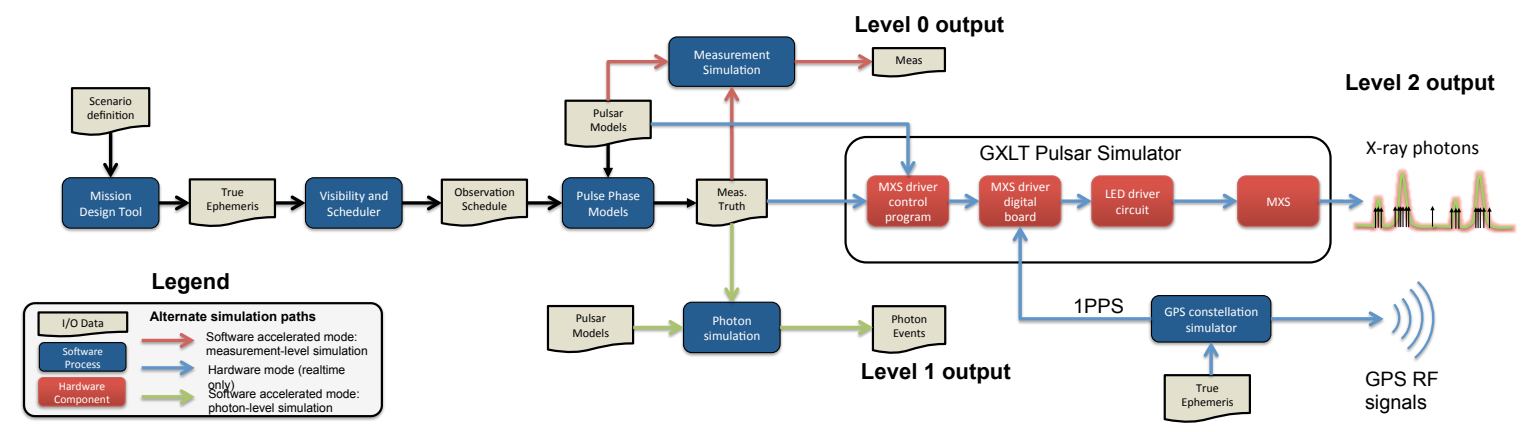

Figure 7. GXLT end-to-end multi-level simulation, showing the flow from orbit simulation through stimulus output.

\section{Baseline Performance and Unit Test}

First, a baseline level of performance is established using the GXLT simulation Level-1 stimulus and a mixed MATLAB/XFSW implementation of the flight software algorithms. The test configuration is shown in Figure 8. There, a MATLAB control program collects batches of photons and runs side-by-side comparisons of MATLAB and XFSW photon processing routines called through a shared library interface to the compiled XFSW, serving as a unit-test of those functions. The control program then sends the output measurements into the XFSW navigation filter functions through the shared library interface. The filter produces a spacecraft orbit estimate which is compared to the truth ephemeris to establish the baseline performance level. This performance level is considered satisfactory if it meets the SEXTANT KPP (10 km accuracy, worst direction). Additional outputs, especially measurement residuals, are also checked to see that they meet expectations. The unit test of the photon processing routines passes if the MATLAB and XFSW photon processing routines give identical results.

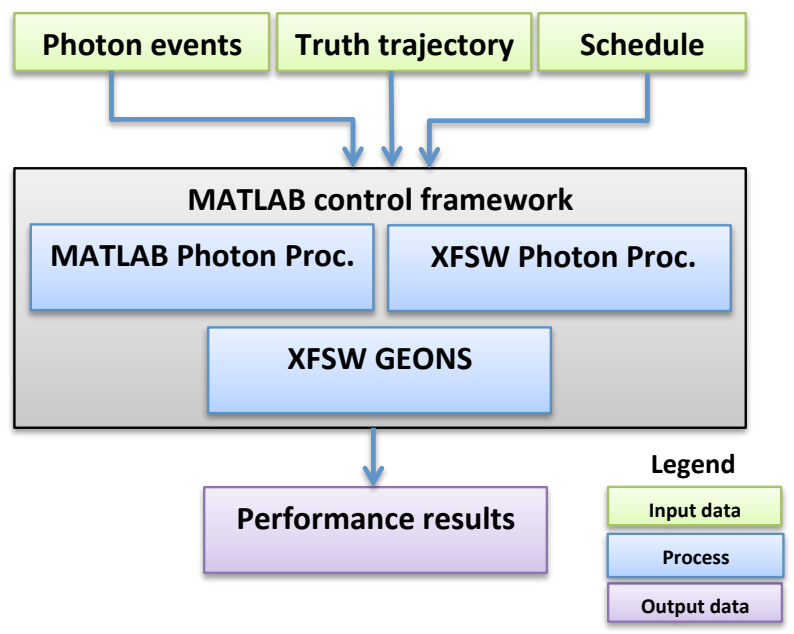

Figure 8. Baseline/Unit test configuration.

Next we present results from the XFSW v2.1.0 build verification testing. Figure 9 shows the Root Sum Square (RSS) position and velocity errors (solid blue lines) relative to the truth ephemeris, along with the $3 \sigma$ filter (root) covariance (dashed red lines), which consistently envelope the errors. Figure 9 shows that the SEXTANT KPP is met with margin: after an initial convergence period, position errors reach

Moving its location in the sky, while not ideal, is not expected to strongly affect performance results, and will be avoided in future tests.
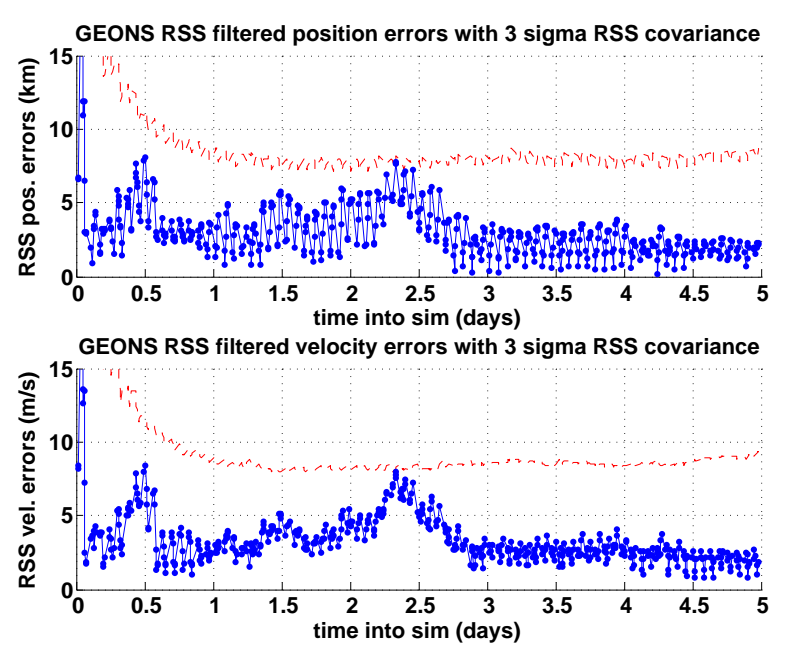

Figure 9. RSS position and velocity errors (solid blue lines) for the baseline/unit-test with enveloping $3 \sigma$ RSS covariance (dashed red lines).

levels well below $10 \mathrm{~km}$ RSS - an upper bound for the "worst direction" referred to by the KPP. The increase in the error between 2.0 and 2.5 days is thought to be random behavior associated with the particular initial state error and simulated photon process. Note the errors in this period remain consistent with the filter covariance. Figure 10 shows the phase measurement residuals (dots) relative to the filter phase predictions, along with the filter's $3 \sigma$ phase prediction variance (solid lines). The actual residual statistics appear to be consistent with the filter variance. The filter is set up to reject any measurement whose residual exceeds the $3 \sigma$ variance. Such rejected measurements are highlighted with a red star mark. The Unit Test also passed, with both MATLAB and XFSW photon processing routines producing identical results (to within expected numerical tolerance).

\section{XFSW App Test}

In the XFSW app test, photon events, schedule data, and truth trajectory information, from the Level-1 GXLT simulation are converted to NICER/SEXTANT Interface Control Document compliant Consultative Committee for Space Data Systems (CCSDS) Photon event, GPS, and Pointing Control packets and sent into a shared library of the full NICER IFSW with integrated XFSW app using a Python interface script. Alternatively, recorded CCSDS packets from a GXLT Level-2 simulation or from flight data can be replayed across this interface. The test configuration is shown in Figure 11. 

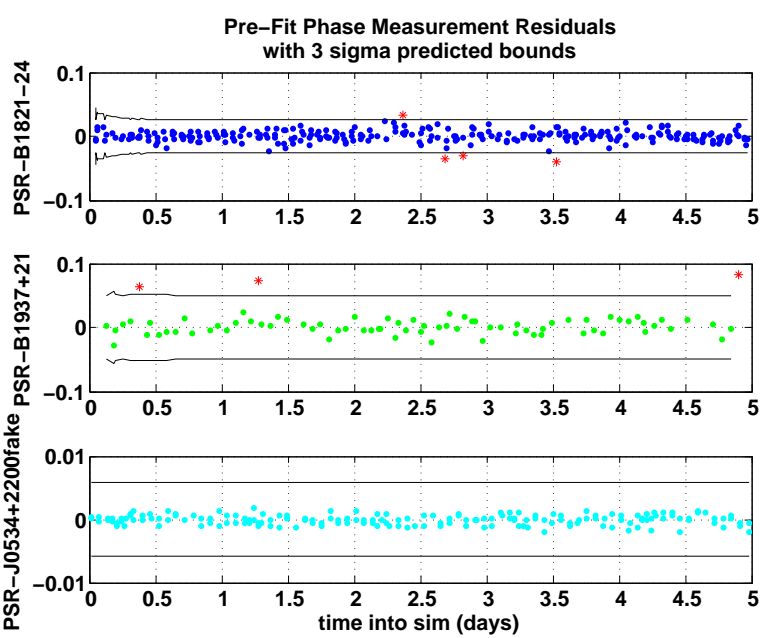

Figure 10. Phase measurement residuals (dots, units of cycles) for baseline/unit-test with $3 \sigma$ covariance (solid lines). Measurements with residuals beyond the $3 \sigma$ level, indicated with a red star mark, are rejected by the filter.

In this test, the XFSW runs on a PC, allowing the use of standard software debugging tools, and is able to run much faster than real-time, both great advantages for development. Code coverage and estimates of the relative computational cost of different routines are obtained. Performance results from this test should be similar to that of the baseline test, with the exception that the measurement times can deviate somewhat due to minor differences in measurement time logic between the MATLAB controlled baseline simulation and the full XFSW app. The test passes if the SEXTANT $\mathrm{KPP}$ is met.

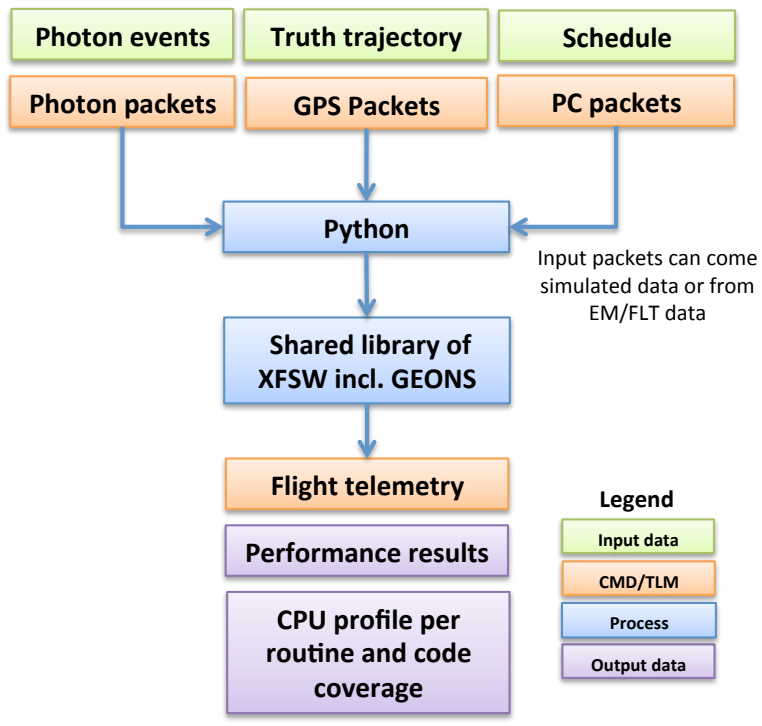

Figure 11. XFSW app test configuration.

Results of the successful XFSW v2.1.0 App Test are presented next. Figure 12 presents the RSS position and velocity errors and corresponds to Figure 9 for the baseline test. In this test, state outputs are provided at the 10 seconds nominal navigation filter propagation step, as opposed to only at the measurement times, which provides more detail than the corresponding baseline test plot. The results are similar to
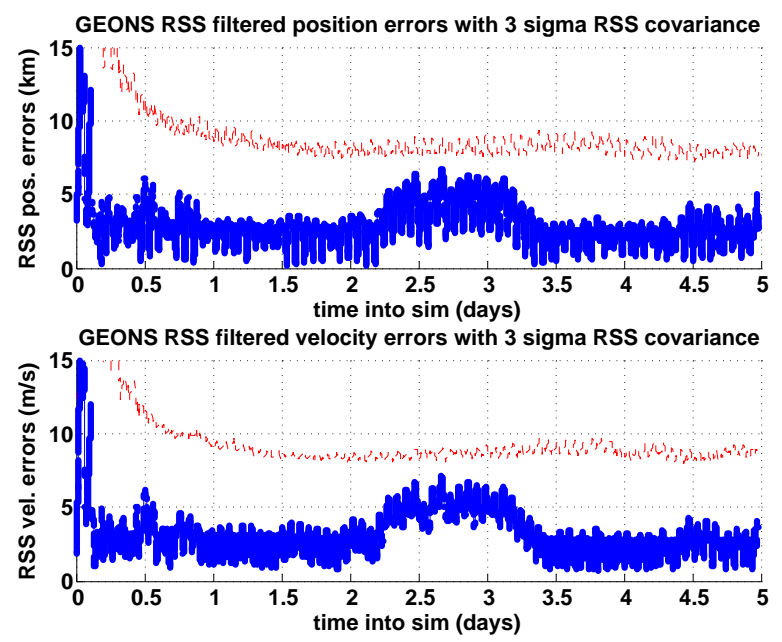

Figure 12. RSS position and velocity errors (solid blue lines) for the XFSW app test with enveloping $3 \sigma$ RSS covariance (dashed red lines).
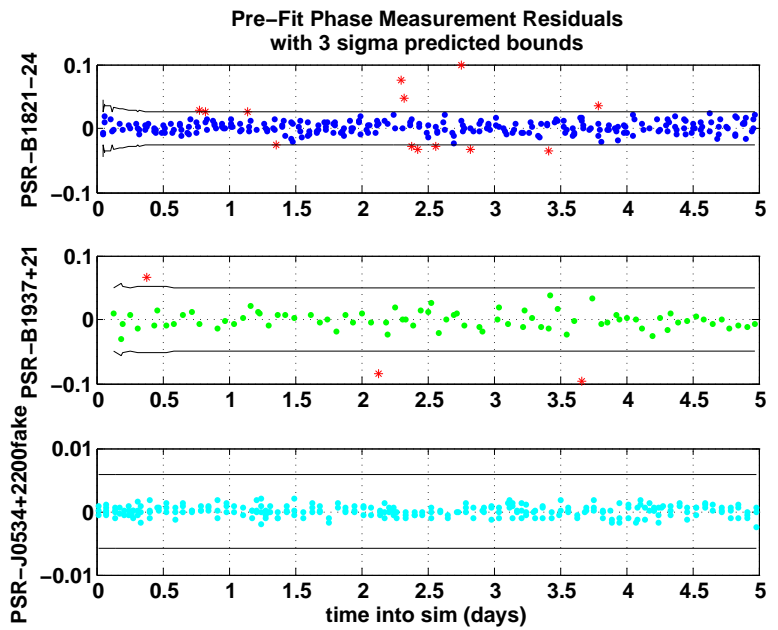

Figure 13. Phase measurement residuals (dots, units of cycles) for XFSW app test with $3 \sigma$ covariance (solid line). Measurements with residuals beyond the $3 \sigma$ level, indicated with a red star mark, are rejected by the filter.

those in the baseline, but not identical. This can be explained by the differences in measurement times and different initial state used here. The increase in the error between 2-3.5 days into the simulation is also present here, persisting longer than in the baseline, but again is consistent with the filter covariance. The SEXTANT KPP is again clearly met in this test. Figure 13 provides the phase measurement observed minus predicted residual and corresponds and appears very similar to Figure 10 from the baseline test.

\section{XFSW Integration Test}

The IFSW Integration Test, shown schematically in Figure 14, is very similar to the XFSW App Test, except that it runs on the NICER EM or Flight processor rather than on a PC, and instead of having the CCSDS packets sent in by a Python script as the test runs, a Python script bundles the input packets into a file of commands that can be uploaded to the flight processor and played back onto the CFS software 
bus using the FileCommander application included in the IFSW. This test validates use of the App Test for longer runs, and provides realistic CPU utilization estimates.

Due to size limitations on the command file, the full rate of the Crab pulsar can only be supported for a maximum of approximately one hour, which is longer than the longest possible uninterrupted observation possible with NICER. Thus, during v2.1.0 build verification testing, two separate tests were conducted to try to achieve coverage of operational modes under this constraint: one long test with the Crab observations removed from the file, and one short test with a limited number of observations of the Crab pulsar. For the former test, we check that the SEXTANT KPP is met and that similar results are obtained as for the Baseline and App Test, however, since it is missing the Crab observations, we expect to achieve somewhat different and possibly degraded performance results. For the latter test, we are primarily interested in seeing that the Crab measurement processing is functional and that measurement residuals are small.

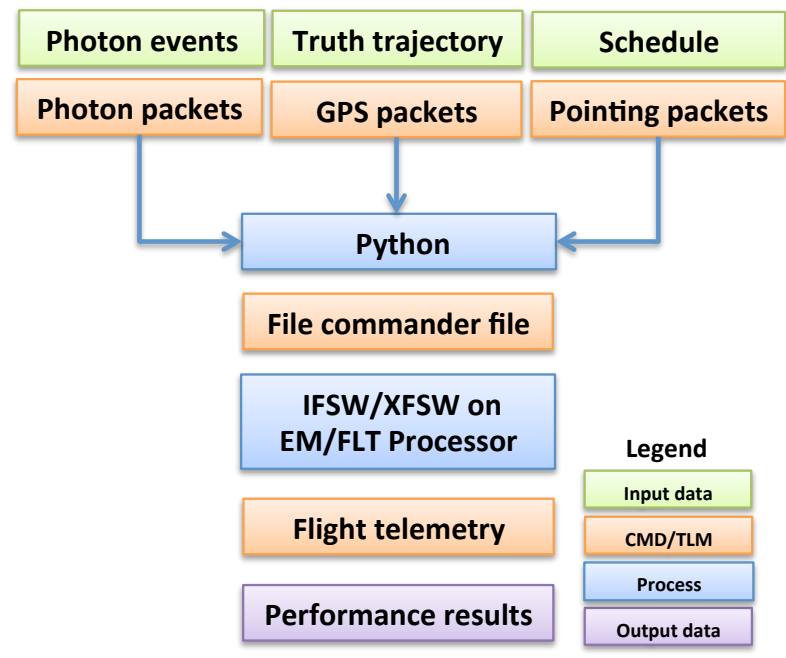

Figure 14. IFSW integration test (no Crab) configuration.

Results for the no-Crab test from XFSW v2.1.0 build verification testing are shown in Figure 15, which gives the RSS errors corresponding to Figure 9 from the baseline test, and Figure 16 gives the phase measurement residuals, corresponding to Figure 10 from the baseline test. Here, even though we omit the Crab measurements, and may expect degraded performance, the RSS error and residual plots are again similar to the baseline test over the reduced simulation time of this test. In particular, the SEXTANT KPP is again met. The Crab test, whose results are not shown here, also passed with small residuals.

\section{Hardware System Test}

The Hardware System Test provides a test-as-you-fly configuration for validation of the SEXTANT flight system. Shown in Figure 17, this test uses the GXLT Level-2 to stimulate the NICER GPS receiver with GPS RF signals modeling the ISS orbit and payload attitude, while providing orbit and schedule modulated X-ray photons to the NICER timing chain, which delivers GPS-derived time-stamps to the XFSW residing within the IFSW running on the NICER EM or Flight processor. Pointing control packets are created from the observation schedule and sent to the IFSW at the appropriate times using an Advanced Spacecraft Integration and System Testing Software (ASIST) ground system software procedure
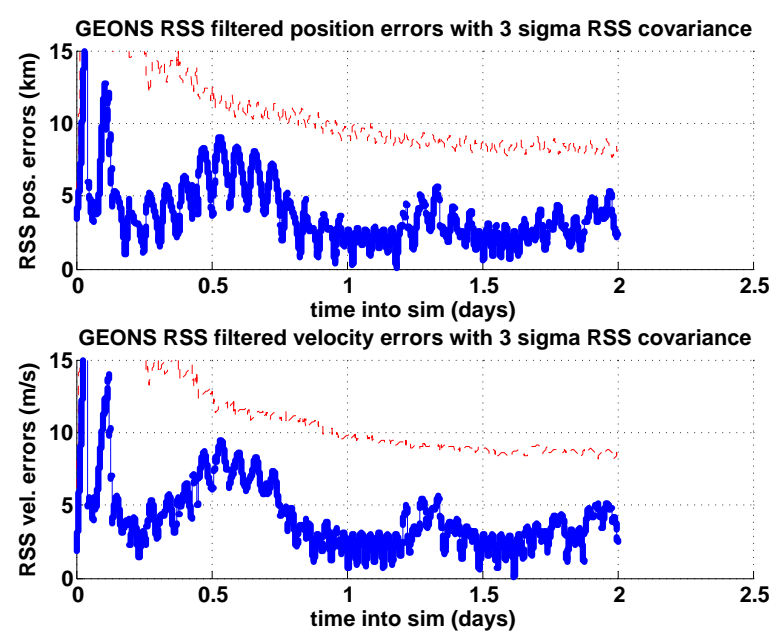

Figure 15. RSS position and velocity errors (solid blue lines) for the IFSW integration test (no Crab) with enveloping $3 \sigma$ RSS covariance (dashed red lines).
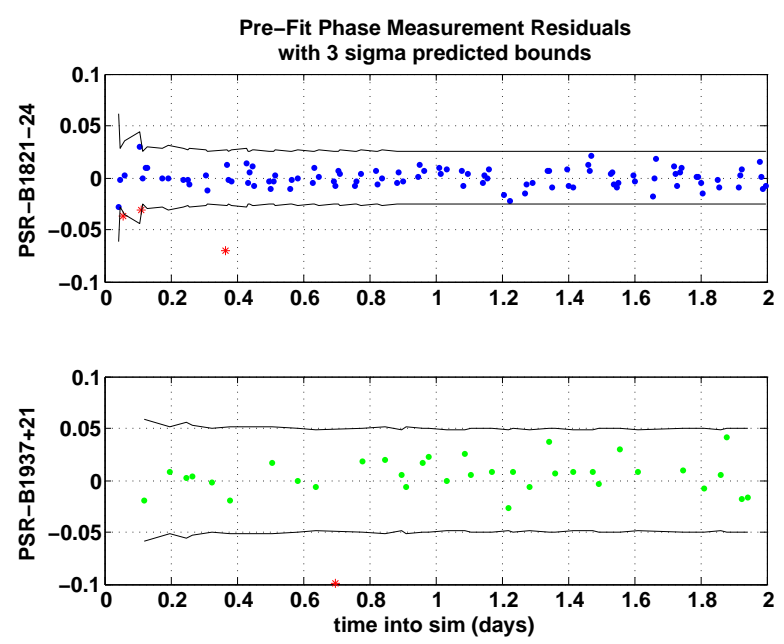

Figure 16. Phase measurement residuals (dots, units of cycles) for IFSW integration test (no Crab) with $3 \sigma$ covariance (solid lines). Measurements with residuals beyond the $3 \sigma$ level, indicated with a red star mark, are rejected by the filter.

timed off the GPS telemetry packets. The XFSW initializes its state estimate using an intentionally degraded GPS state and maintains it with XNAV measurements. Performance of the system is monitored by the XFSW in real-time by comparing to GPS states, as planned on-orbit.

For v2.1.0 build verification testing, the Hardware System Test was run on the NICER MEB EM. GPS RF stimulus was provided using a Spirent GPS constellation simulator running an high-fidelity ISS orbit scenario for 3.5 days. The pulsar simulator was set up to stimulate a single-string EM NICER timing chain consisting of a single Focal Plane Module and Measurement/Power Unit (MPU) connected to the MEB. In addition to the use of the fake Crab, due to limited dynamic range of the GXLT Pulsar simulator, the total count rate for the Crab was reduced to a similar level as the other MSPs which were set to a common level of approximately 100 counts per second impinging on the detector. Within the XFSW, all Crab photons (passing filter criteria) were 
accepted, but a photon-by-photon random filter was used to keep only a small fraction of the events for the other Pulsars. The effect was to achieve approximately 100 counts per second for the Crab, and accurate total and relative count rates for the remaining Pulsars. While this is a factor of approximately 2 lower flux than what is expected on-orbit for the Crab, all algorithmic aspects of Crab processing were still exercised. Significantly higher count rates, but still short of on-orbit expectations, will be achieved in future hardware system ground tests. Finally, we note that the signal to noise ratio was significantly elevated in this test. This was due to a nonlinearity in the Modulated X-Ray Source (MXS) Light Emitting Diode (LED) output intensity vs. input current characteristic which resulted in suppressed background rates and increased signal-to-noise ratio. This effect will be compensated for in subsequent build testing.

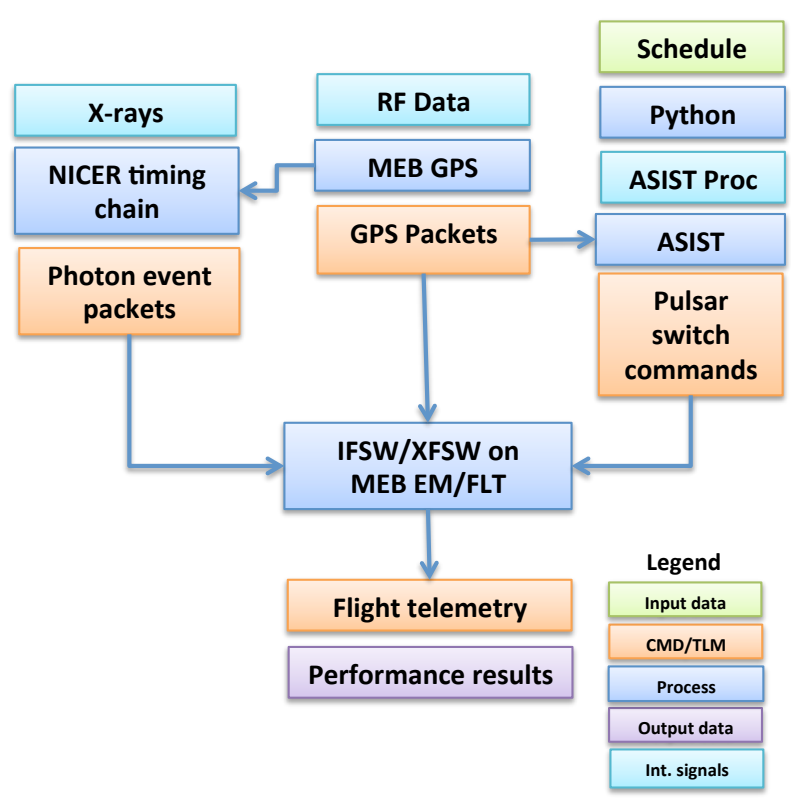

Figure 17. Hardware System Test configuration.

RSS error results from the v2.1.0 Hardware System Test are shown in Figures 18, while the phase residuals are shown in Figure 19. Not surprisingly, due to the elevated signal-tonoise ratio achieved in this test, and discussed above, the RSS error performance is significantly better for Hardware System Test as compared to that of the baseline test (Figure 9), with the SEXTANT KPP again being met. The residual plot again shows consistency between the actual residuals and the filter's residual variance estimate. We note the glitches in the RSS error plots-one for the actual error around 1.25 days into the simulation, and one on each plot for the covariance at around 2.2 days into the simulation. These are thought to be caused by an issue with the telemetry stream and not related to actual performance.

\section{Conclusions}

NASA's SEXTANT project, a technology enhancement to the ISS-bound NICER mission [3, 4], aims to provide the first demonstration of autonomous spacecraft navigation using $\mathrm{X}$ ray pulsars or $X N A V$. If successful, this demonstration will provide a major step toward enabling a GPS-like autonomous navigation capability available throughout the solar system and beyond.
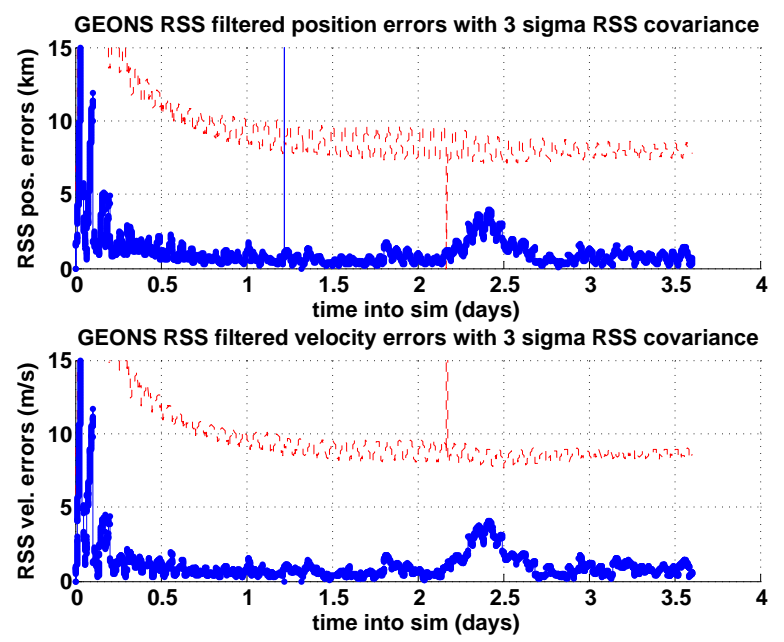

Figure 18. RSS position and velocity errors (solid blue lines) for the Hardware System Test with enveloping $3 \sigma$ RSS covariance (dashed red lines). The spikes in the error are believed to be telemetry collection and/or post-processing issues, not related to actual performance.
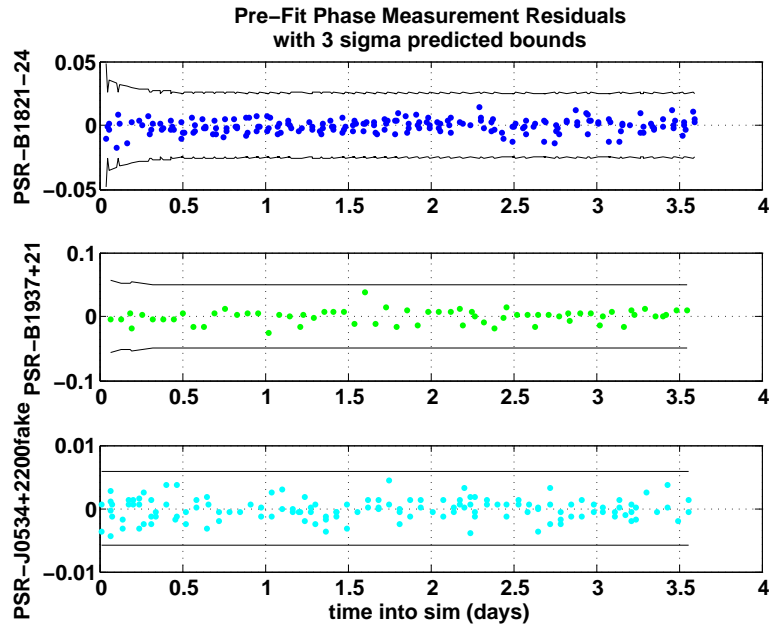

Figure 19. Phase measurement residuals (dots, units of cycles) for Hardware System Test with $3 \sigma$ covariance (solid lines).

This paper serves as a companion to previous publications [7] and [2], where the SEXTANT architecture, algorithms, and testbed were described. Here, we provide an update to the SEXTANT algorithms described in [2] and give first detailed descriptions of the SEXTANT flight system consisting of the XFSW and XGS. Finally, we provide a detailed description of the XFSW build verification test suite, which runs in the high-fidelity GXLT, and present results from the successful testing of build 2.1.0.

Beyond verifying the basic functionality and stability of XFSW build 2.1.0, the test results suggest that the SEXTANT flight system is on target to meet its key performance goal of maintaining better than $10 \mathrm{~km}$ (worst direction) definitive orbit knowledge using only XNAV measurements. Additional tests using the GXLT will be conducted on v2.1.0, and future builds, to address some of the limitations of the build test suite that are discussed in this paper. These tests will include 
comprehensive Monte Carlo trials and stress tests to attempt to establish margin for meeting performance goals in the face of the many unknowns of the true on-orbit environment.

SEXTANT completed its annual continuation review in September 2015 and is progressing on-schedule toward a final flight system implementation. XFSW v2.1.0 was integrated into the most recent NICER Instrument Flight Software build and is supporting payload testing. The final XFSW build is planned for early 2016. The XGS architecture and basic operations concept is defined, with deployment of a major build planned in late 2015 and a final build planned for mid 2016. NICER is progressing through its integration and test phase and is on-target for an August 2016 launch via SpaceX Commercial Resupply Services mission.

\section{ACKNOWLEDGMENTS}

The authors would like to thank Dr. Russell Carpenter for his valuable input and time.

This research has made use of data, software and/or web tools obtained from NASA's High Energy Astrophysics Science Archive Research Center, a service of Goddard Space Flight Center and the Smithsonian Astrophysical Observatory.

\section{BIOGRAPHY}

Dr. Luke M.B. Winternitz is an engineer at NASA GSFC in the Components and Hardware Systems branch. He is the SEXTANT Systems Architect and the technical lead of the space-GPS receiver research and development group. He received his Ph.D. (2010) in Electrical Engineering from the University of Maryland, College Park.

Dr. Jason W. Mitchell is an engineer at NASA GSFC in the Navigation and Mission Design branch, and is the project manager for the Station Explorer for X-ray Timing and Navigation Technology (SEXTANT). He received his Ph.D. (2000) in Aerospace Engineering from the University of Cincinnati.

Dr. Munther A. Hassouneh is an engineer at NASA GSFC in the Components and Hardware Systems branch. He is the SEXTANT ground testbed lead and is a member of the space-GPS receiver research and development group. He received his Ph.D. (2003) in Electrical Engineering from the University of Maryland, College Park.

Ms. Jennifer E. Valdez is an engineer at NASA GSFC in the Components and Hardware Systems branch. She is the SEXTANT lead for the XNAV end-to-end simulator and is a member of the space-GPS receiver research and development group.

Mr. Wayne H. Yu Mr. Wayne H. Yu is an engineer at NASA GSFC in the Navigation and Mission Design Branch. He performs navigation and flight dynamics analysis for the XNAV end-to-end simulator, focusing on visibility and observation schedule modeling. He received his M.S. (2015) in Aerospace Engineering from the University of Maryland, College Park.

Dr. Sean R. Semper is an engineer at NASA GSFC in the Components and Hardware Systems branch and he is the SEXTANT ground system lead. He received his Ph.D. (2011) in Aerospace Engineering from the University at Buffalo, SUNY.

Dr. Paul S. Ray is an astrophysicist at the Naval Research Laboratory. He is a NICER science team member specializing in high precision timing observations of pulsars. He received his Ph.D. (1995) in Physics from the California Institute of Technology.

Dr. Kent S. Wood is an astrophysicist at the Naval Research Laboratory. He is a NICER science team member specializing in pulsar observations and observations involving other celestial source classes, as well as calibrations and background modeling. He received his Ph.D. (1973) in Physics from the Massachusetts Institute of Technology.

Dr. Zaven Arzoumanian is a Senior Research Scientist with the Universities Space Research Association (USRA), working within the Center for Research and Exploration in Space Science and Technology (CRESST) at NASA's Goddard Space Flight Center. He currently serves as Deputy Principal Investigator and Science Lead for NICER.

Dr. Keith C. Gendreau is currently the principal investigator of the X-ray Advanced Concepts Test Bed sounding rocket, the SEXTANT demonstration, and the NICER mission. He received his Ph.D. (1995) in Astrophysics from the Massachusetts Institute of Technology, and was the NASA scientist on the joint NASA-DARPA $X$-ray pulsar source-based navigation and timing study.

\section{REFERENCES}

[1] J. W. Mitchell, M. A. Hassouneh, L. M. Winternitz, J. E. Valdez, S. R. Price, S. R. Semper, W. H. Yu, Z. Arzoumanian, P. S. Ray, K. S. Wood, R. J. Litchford, and K. C. Gendreau, "SEXTANT - Station Explorer for X-ray Timing and Navigation Technology," in AIAA Guidance, Navigation, and Control Conference, ser. AIAA SciTech. American Institute of Aeronautics and Astronautics, Jan. 2015. [Online]. Available: http://dx.doi.org/10.2514/6.2015-0865

[2] L. Winternitz, M. Hassouneh, J. Mitchell, J. Valdez, S. Price, S. Semper, W. Yu, P. Ray, K. Wood, Z. Arzoumanian, and K. Gendreau, "X-ray pulsar navigation algorithms and testbed for SEXTANT," in Aerospace Conference, 2015 IEEE. IEEE, March 2015. [Online]. Available: http://dx.doi.org/10.1109/ AERO.2015.7118936

[3] K. C. Gendreau, Z. Arzoumanian, T. Okajima, and the NICER Team, "The Neutron-star Interior Composition ExploreR (NICER): an Explorer mission of opportunity for soft X-ray timing spectroscopy," in Space Telescopes and Instrumentation: Ultraviolet to Gamma Ray, ser. Proc. SPIE, vol. 8443. International Society for Optics and Photonics, Sep 2012. [Online]. Available: http://dx.doi.org/10.1117/12.926396

[4] Z. Arzoumanian, K. C. Gendreau, C. L. Baker, 
T. Cazeau, P. Hestnes, J. W. Kellogg, S. J. Kenyon, R. P. Kozon, K.-C. Liu, S. S. Manthripragada, C. B. Markwardt, A. L. Mitchell, J. W. Mitchell, C. A. Monroe, T. Okajima, S. E. Pollard, D. F. Powers, B. J. Savadkin, L. B. Winternitz, P. T. Chen, M. R. Wright, R. Foster, G. Prigozhin, R. Remillard, and J. Doty, "The Neutron-star Interior Composition Explorer (NICER): Mission Definition," in Astronomical Telescopes and Instrumentation, ser. Proc. SPIE, vol. 9144. International Society for Optics and Photonics, 2014, pp. 914420-914420-9. [Online]. Available: http://dx.doi.org/10.1117/12.2056811

[5] A. Long, "Goddard Enhanced Onboard Navigation System (GEONS) Mathematical Specifications," a.i. solutions Inc., Tech. Rep. FDSS-23-0035, November 2012, Version 2, Release 2.17.

[6] _ - "Goddard Enhanced Onboard Navigation System (GEONS) System Description and User's Guide," a.i. solutions Inc., Tech. Rep. FDSS-23-0026, November 2012, Version 2, Release 2.17.

[7] J. W. Mitchell, M. A. Hassouneh, L. B. Winternitz, J. E. Valdez, P. S. Ray, Z. Arzoumanian, and K. C. Gendreau, "Station Explorer for X-ray Timing and Navigation Technology Architecture Overview," in Proceedings of the 27th International Technical Meeting of the Satellite Division of the Institute of Navigation (ION GNSS+). Institute of Navigation, 2014.

[8] NASA, "Systems Engineering Handbook," National Aeronautics and Space Administration, Washington, DC, USA, Tech. Rep. NASA/SP-2007-6105, December 2007, Rev 1. [Online]. Available: http://go.usa.gov/ $3 \mathrm{tV} 8 \mathrm{C}$

[9] J. Nocedal and S. J. Wright, Numerical Optimization, 2nd ed. New York: Springer, 2006.

[10] J. Wilmot, "A Core Flight Software System," in Proceedings of the 3rd IEEE/ACM/IFIP International Conference on Hardware/Software Codesign and System Synthesis, ser. CODES+ISSS '05. New York, NY, USA: ACM, 2005, pp. 13-14. [Online]. Available: http://doi.acm.org/10.1145/1084834.1084842

[11] A. Nicholas, "Miniature Array of Radiation Sensors (MARS)," August 2015, Accessed: 2015-09-23. [Online]. Available: http://go.usa.gov/3tpcm

[12] R. T. Edwards, G. B. Hobbs, and R. N. Manchester, "TEMPO2, a new pulsar timing package - II. the timing model and precision estimates," Monthly Notices of the Royal Astronomical Society, vol. 372, no. 4, pp. 15491574, 2006, pre-print: arXiv:astro-ph/0607664.

[13] L. B. Winternitz, M. A. Hassouneh, J. W. Mitchell, F. Gavriil, Z. Arzoumanian, and K. C. Gendreau, "The Role of X-rays in Future Space Navigation and Communication," in 36th Annual Guidance \& Navigation Control Conference. American Astronautical Society, February 2013.

[14] K. M. Getzandanner, "Orbit Determination Toolbox (ODTBX)," NASA Goddard Space Flight Center Navigation and Mission Design Branch, 2012, retrieved Sep 12, 2012. [Online]. Available: http://odtbx.sf.net/

[15] S. P. Hughes, "General Mission Analysis Tool (GMAT)," NASA Goddard Space Flight Center Navigation and Mission Design Branch, 2012, retrieved Sep 6 2012. [Online]. Available: http: //gmat.gsfc.nasa.gov/ 